\title{
Comparative analysis of biotin intranuclear inclusions of gestational endometrium using the APAAP, ABC and the PAP immunodetection systems
}

\author{
K Cooper, Z Haffajee, L Taylor
}

\begin{abstract}
Aims/background-Intranuclear inclusions have been observed in gestational endometrial glands. Although resembling Herpes simplex virus infected cells, these nuclei have been shown to contain endogenous biotin. Hence, immunohistochemical systems using the avidin-biotin complex will result in cross reaction and false positivity. This study investigates a series of 10 gestational endometria (formalin fixed and paraffin wax embedded) with intranuclear inclusions using three different immunodetection systems with primary anti-biotin.
\end{abstract}

Results-Both the alkaline phosphatase anti-alkaline phosphatase (APAAP) and peroxidase anti-peroxidase (PAP) systems confirmed that the intranuclear inclusions were endogenous biotin. The streptavidin biotin complex (StreptABC) system produced a positive reaction in both test sections and controls (omitting primary anti-biotin) in the presence of prior blocking with free avidin and biotin. In addition, aberrant immunoreactivity was observed in adjacent nuclei/cytoplasm of endometrial glands and decidualised stroma in the negative control of the PAP system. This was subsequently eliminated using microwave pretreatment prior to immunohistochemistry.

Conclusion-The use of either the APAAP or PAP immunodetection systems (the latter with microwave pretreatment) is recommended for any immunohistochemical or non-isotopic in situ hybridisation investigation undertaken on gestational endometria.

(f Clin Pathol 1997;50:153-156)

Anatomical Pathology,

School of Pathology,

University of

Witwatersrand and

South African Institute

for Medical Research,

Johannesburg, South

Africa

K Cooper

Z Haffajee

L Taylor

Correspondence to:

Professor K Cooper,

Department of Anatomical

Pathology, SAIMR, PO Box

1038, Johannesburg 2000,

South Africa.

Accepted for publication

5 November 1996

Keywords: gestational endometrium; alkaline phosphate anti-alkaline phosphatase; avidin-biotin complex; peroxidase anti-peroxidase; in situ hybridisation.

Intranuclear inclusions have been occasionally observed in endometrial glands, particularly during gestation and puerperium. These optically clear nuclei with a ground-glass appearance resemble Herpes simplex virus (HSV) infected cells. ${ }^{2}$ However, these inclusions contain biotin, as shown immunohistochemically using monoclonal anti-biotin as the primary antibody and the peroxidase antiperoxidase (PAP) method for detection. ${ }^{12}$
Biotin is a co-enzyme that participates in gluconeogenesis and fatty acid biosynthesis. Endogenous biotin is present in fairly large amounts in kidney, liver, pancreas, mammary gland, adipose tissue, and skeletal muscle, ${ }^{34}$ and has been reported recently in pregnancy related endometria. ${ }^{12}$

Previous researchers have recommended blocking endogenous biotin with $0.05 \%$ free avidin and $0.05 \%$ free biotin to abolish cross reaction with endogenous biotin when using the streptavidin biotin complex/horseradish peroxidase (StreptABC/HRP) method. ${ }^{2}$ An alternate recommendation to abolish endogenous biotin in gestational endometria suggested the use of a detection system, such as the PAP method, which would avoid the avidin/ biotin blocking procedure. $^{2}$

This study evolved from a case that was routinely investigated for HSV (Biomeda, USA) using immunohistochemistry. The sections were pre-incubated with free avidin $(0.1 \%)$ and free biotin $(0.01 \%)$ (Dako, Glostrup, Denmark) prior to immunodetection for $\mathrm{HSV}$ using the StreptABC/HRP method as recommended by the manufacturer (Dako Biotin blocking system). A negative control was included using a parallel section in which the primary antibody was omitted. Despite using the blocking procedure, positive intranuclear immunoreaction was noted in the negative control, prompting this study.

\section{Methods}

Ten cases of gestational endometrial curettings were retrieved from the archives of the Department of Anatomical Pathology. All tissue had been fixed in formalin and embedded in paraffin wax. Cases were included in the study if intranuclear ground-glass inclusions were present within endometrial glands. The HIV status of these patients was negative.
All biopsy specimens were examined immunohistochemically using monoclonal antibiotin (Dako) as the primary antibody, followed by the ABC method, the PAP complex method and the alkaline phosphatase antialkaline phosphatase method (APAAP), for the detection of endogenous biotin intranuclear inclusions.

\section{IMMUNODETECTION SYSTEMS}

$A B C$ method

Sections were dewaxed and rehydrated. Endogenous peroxidase was quenched using 3\% 

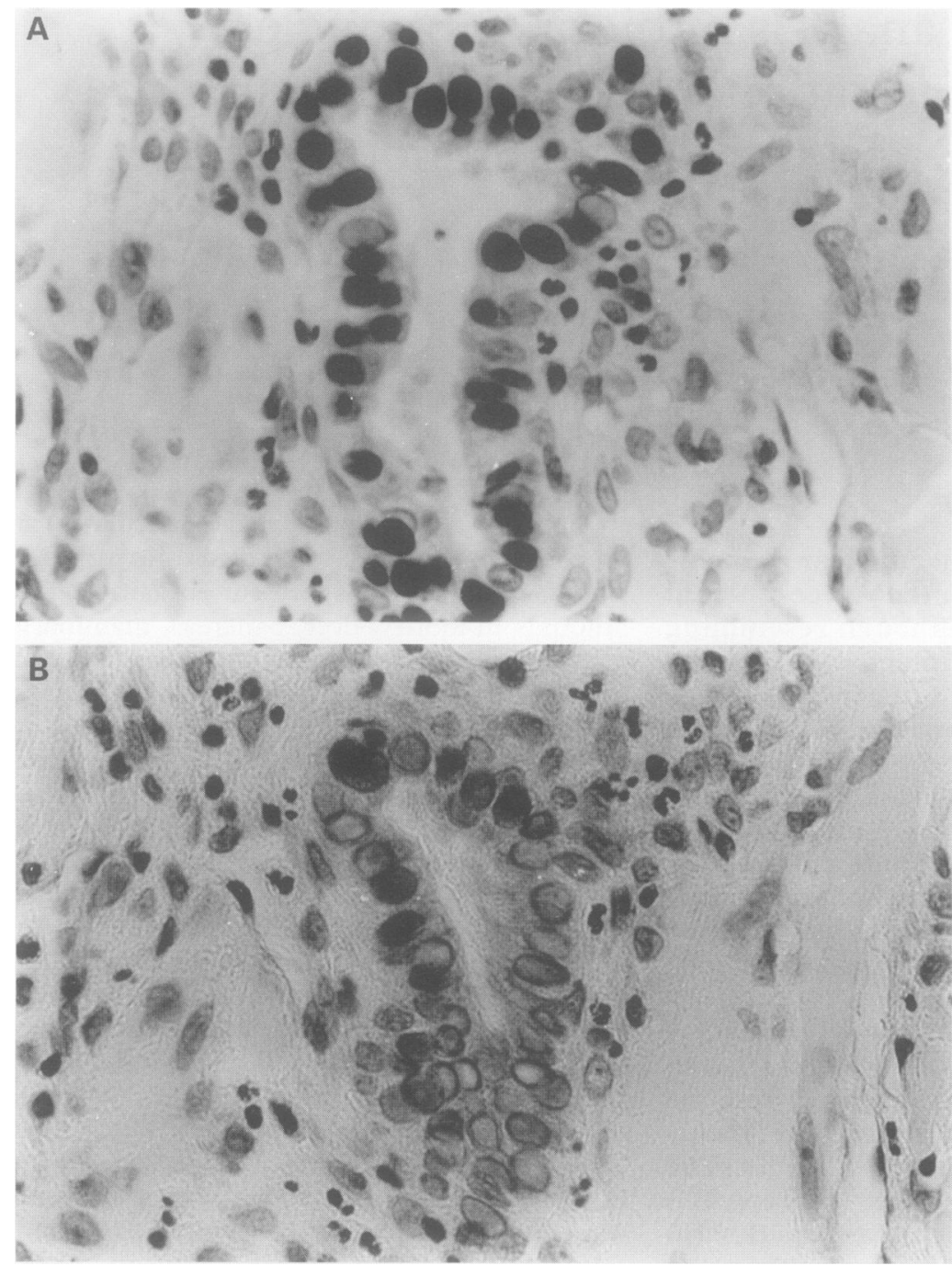

Figure $1 A B C$ system. Intranuclear inclusions show immunopositivity, $(A)$ with and $(B)$ without (albeit lighter) primary anti-biotin, in gestational endometrial glands after blocking endogenous biotin with free avidin/free biotin.

hydrogen peroxide in methanol for 30 minutes. All sections were pre-incubated with $0.1 \%$ avidin for 10 minutes, rinsed in phosphate buffered saline (PBS) ( $\mathrm{pH} \mathrm{7.6)} \mathrm{and} \mathrm{re-}$ incubated with $0.01 \%$ biotin for 10 minutes, then washed in PBS. The sections were then incubated with $5 \%$ normal horse serum for 20 minutes, monoclonal anti-biotin at a 1 in 20 dilution for 30 minutes, biotinylated secondary horse-anti-mouse IgG antibody for $30 \mathrm{~min}$ utes, avidin/biotin complex for 30 minutes, and finally $0.1 \%$ diaminobenzidine tetrahydrochloride (DAB) $/ 0.03 \%$ hydrogen peroxide for five minutes. Mayer's haematoxylin was used as the counterstain. The sections were then dehydrated and mounted in Entellen.

\section{APAAP method}

Sections were dewaxed, rehydrated, and then incubated with normal rabbit serum (diluted 1 in 5) for 20 minutes, monoclonal anti-biotin (diluted 1 in 20) for 30 minutes, secondary rabbit-anti-mouse IgG for 30 minutes, APAAP kit (mouse) (Dako) for 30 minutes, and finally with substrate solution-alkaline phosphatase containing naphthol AS-MX phosphate/Fast Red TR as the chromogen. Mayer's haematoxylin was used as the counterstain and the sections were mounted in an aqueous medium (Glycergel; Dako).

\section{PAP method}

Sections were dewaxed, rehydrated and quenched as for the $A B C$ method, and then incubated with normal rabbit serum (diluted 1 in 5) for 20 minutes, monoclonal anti-biotin (diluted 1 in 20) for 30 minutes, rabbit-antimouse IgG secondary antibody for 30 minutes, PAP (rabbit) for 30 minutes, and finally with $\mathrm{DAB}$ as described for the $\mathrm{ABC}$ method. In addition, sections were rinsed in $\mathrm{PBS} /$ bovine serum albumin (BSA) for $2 \times 5$ minutes between every step.

Negative controls omitting the primary antibody were included, using normal serum as a substitute for the primary antibody in all three immunodetection systems.

\section{Results}

ABC METHOD

Biotin was detected in the inclusions in all 10 cases (fig 1) even though endogenous biotin was blocked with free avidin/biotin as recommended previously. ${ }^{2}$ A positive immunoreaction was also seen in the negative control sections, coinciding with the intranuclear inclusions.

\section{APAAP METHOD}

This system was used as an alternative detection system, with monoclonal anti-biotin as the primary antibody to eliminate false positivity resulting from excess endogenous peroxidase or biotin. Endogenous biotin was detected within the intranuclear inclusions of the endometrial glands in all 10 cases (fig 2). The negative control sections were free of any nonspecific staining in the endometrial glands and decidualised stroma.

\section{PAP METHOD}

Biotin was detected in all 10 cases within intranuclear inclusions in endometrial glands. Biotin was not detected within the endometrial glandular intranuclear inclusions in any of the negative control sections, in which anti-biotin was omitted.

However, two of the 10 cases showed aberrant positive staining in the negative controls. This positive reaction was present in the nuclei and cytoplasm of adjacent endometrial glandular epithelium without intranuclear inclusions (fig 3). Occasional decidual stromal cells also showed a similar aberrant immunoreaction within nuclei and cytoplasm. These two cases were then studied using a dual PAP/ APAAP immunohistochemical detection system in which the primary antibody, anti-biotin, was omitted in the initial PAP procedure. After washing in PBS, sections were then immunostained using the APAAP method with monoclonal anti-biotin as the primary antibody. This double PAP/APAAP system did indeed show that the aberrant PAP positive cells were distinctly separate from the APAAP positive, endogenous biotin-containing intranuclear inclusions. 

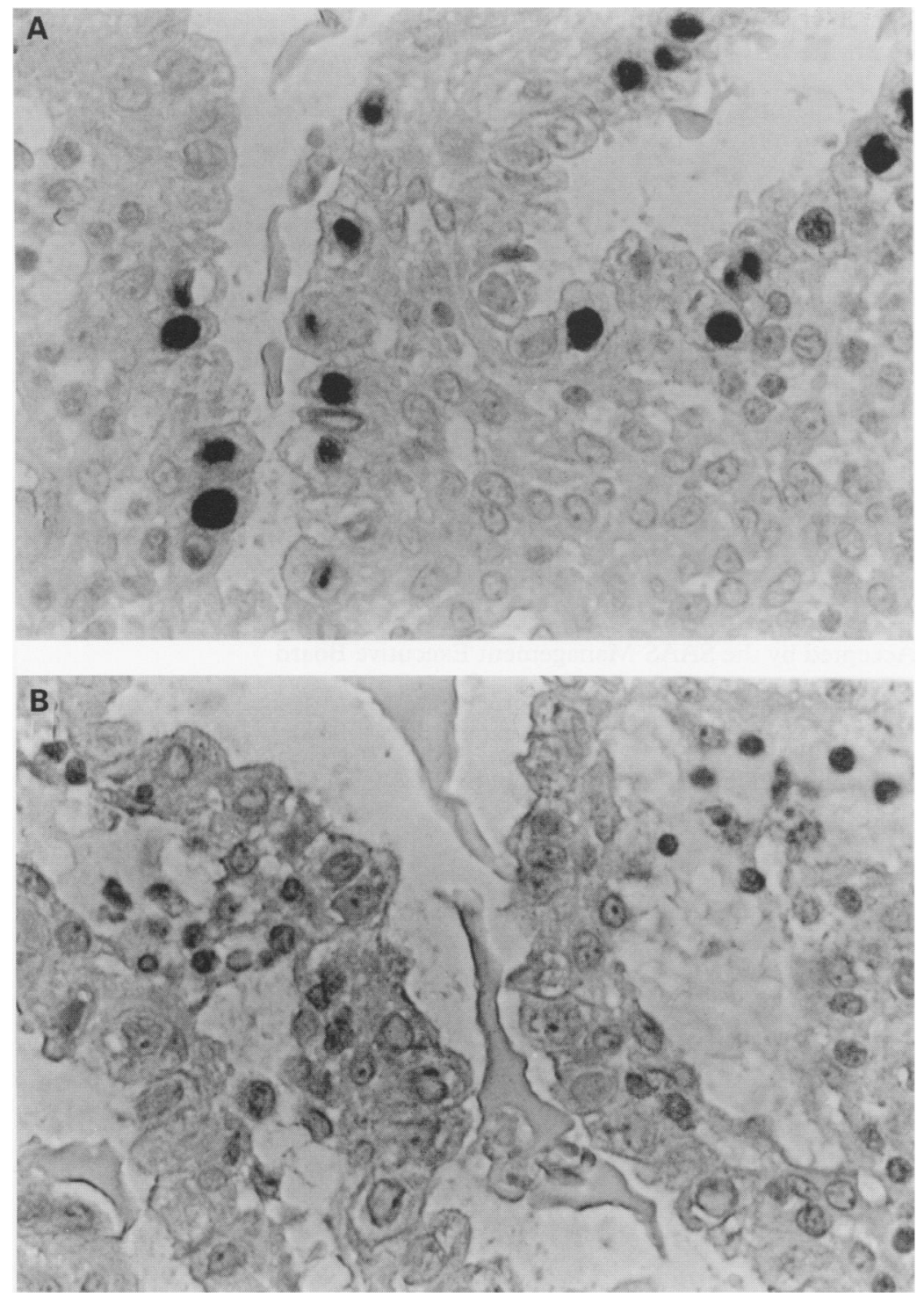

Figure 2 APAAP system. (A) Positive immunoreaction for biotin within intranuclear inclusions. (B) Negative control sections were free of any non-specific staining reaction.

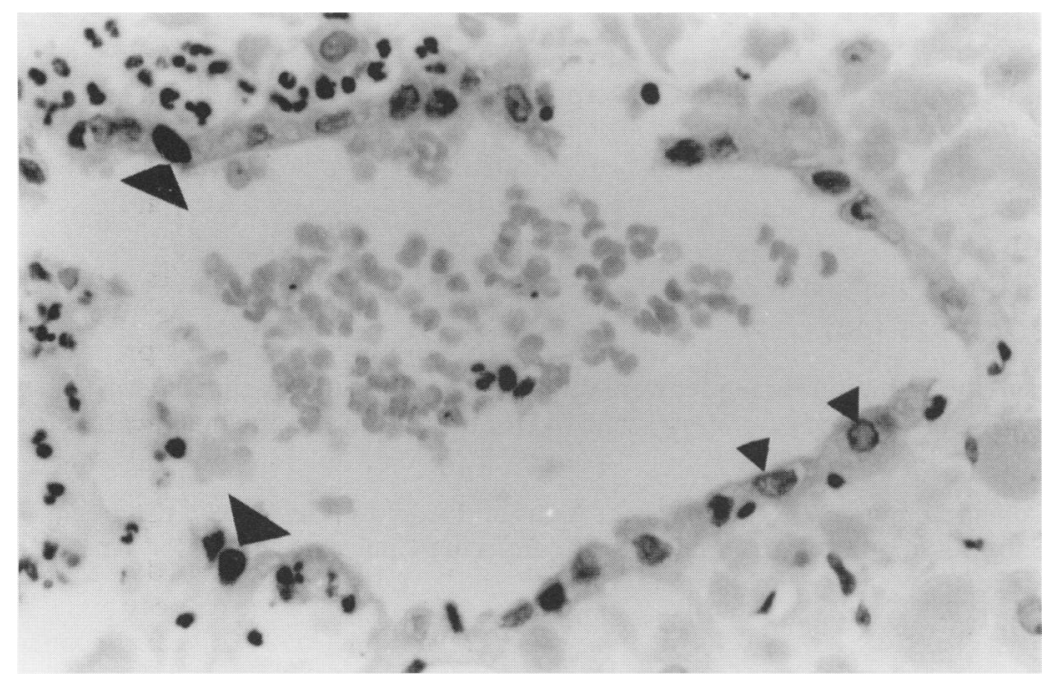

Figure 3 PAP system. Aberrant positive immunoreaction in endometrial glands without intranuclear inclusions in negative control sections (large arrows). This was subsequently eliminated with microwave pretreatment. Note the adjacent intranuclear inclusions are negative (small arrows).

The two cases with the aberrant immunoreaction with the PAP detection system without primary anti-biotin were investigated further. Sections were pretreated in a microwave oven ${ }^{5}$ in $0.01 \mathrm{M}$ citric acid buffer ( $\mathrm{pH} 6.0)(2 \times 5$ minutes) at 700 watts, were allowed to cool in buffer for 25 minutes, and then rinsed in PBS. The PAP method was repeated, again without primary antibody. The non-specific immunoreaction seen in the cytoplasm/nuclei of endometrial glands (without intranuclear inclusions) and adjacent decidualised stroma was completely eliminated.

\section{Discussion}

Intranuclear inclusions in gestational/puerperal endometrial glands resemble HSV infection with peripheral margination of chromatin and central clearing of nuclei producing a groundglass appearance. ${ }^{6}$ This morphological appearance may quite easily be mistaken for HSV infection. However, these inclusions have recently been shown to contain biotin. ${ }^{12}$ Hence, false positivity when using the StreptABC/HRP immunodetection system is a very real problem for the unwary. ${ }^{78}$ By blocking endogenous biotin with free avidin and free biotin, ${ }^{2}$ it was anticipated that the false positivity associated with the StreptABC/HRP immunodetection system would be abolished. In our study, all 10 cases with intranuclear inclusions in gestational endometrial glands showed positive immunoreaction with the StreptABC/HRP system, with and without primary anti-biotin, even when endogenous biotin was quenched. This clearly shows that the StreptABC/HRP system is unreliable in the presence of endogenous biotin.

Our study also confirmed previous studies showing that the gestation-associated endometrial intranuclear inclusions are in fact endogenous biotin. This was accomplished using anti-biotin with two independent immunodetection systems (APAAP and PAP) that are related to neither avidin nor biotin. The use of the APAAP system has not been reported previously in this context. Surprisingly, however, aberrant staining in the nuclei and cytoplasm of adjacent endometrial glands and the decidual stromal cells was observed with the PAP system. This was also the case in the negative control sections without primary anti-biotin. These false positive cells seen with the PAP system could be clearly distinguished from the cells with biotin-containing intranuclear inclusions with the dual PAP/APAAP system. Further investigation using microwave pretreatment with citric acid buffer totally eliminated the aberrant false positivity in the PAP negative controls. Therefore, we recommend that studies on gestation/puerperal endometria using immunohistochemistry and in situ hybridisation should either use the APAAP or PAP immunodetection systems, the latter incorporating microwave pretreatment. Both systems avoid the problems related with endogenous biotin.

The aberrant false positivity associated with the negative control of the PAP system reaffirms the need for negative controls on all sections in every immunohistochemical run. Nevertheless, further studies with a variety of tissues, fixatives and pretreatment systems are warranted to 
investigate the as yet uncharacterised aberrant false positivity associated with the PAP system.

This study was supported with grants awarded to $\mathrm{KC}$ from SAIMR, CANSA and MRC. We are grateful to Mrs Molly Long for preparing the manuscript.

1 Shigeo Y, Kenji K, Souichi I, Daa T, Nakayama I, Moriuchi A. Biotin-containing intranuclear inclusions in endometrial
glands during gestation and puerperium. Am $\mathcal{f}$ Clin Pathol glands during gesta

2 Sickel JZ, di Sant'Agnese A. Anomalous immunostaining of 'optically clear' nuclei in gestational endometrium. Arch Pathol Lab Med 1994;118:831-3.

3 Dakshinamurti K, Mistry SP. Tissue and intracellular distribution of biotin-COOH in rats and chicks. $f$ Biol Chem 1963;238:294-6.
4 Wood GS, Warnke R. Suppression of endogenous avidinbinding activity in tissues and its relevance to biotin-avidin detection systems. F Histochem Cytochem 1982;29:1196204.

5 Cooper K, Haffajee Z. Immunohistochemical assessment of MIC2 gene product in granulocytic sarcoma using six epitope retrieval system. Appl Immunohistochem 1995:3 198-201.

6 Mazur MT, Hendrickson MR, Kempson RL. Optically clear nuclei-an alteration of endometrial epithelium in the presence of trophoblast. Am f Surg Pathol 1983;7:41523.

7 Wright CA, Haffajee Z, Iddekinge B, Cooper K. Detection of Herpes simplex virus DNA in spontaneous abortions from HIV-positive women using non-isotopic in situ from HIV-positive women using non-i
hybridization. $\mathcal{F}$ Pathol 1995;176:399-402.

8 Cooper K. Biotin intranuclear inclusions in gestational endometria [letter]. F Pathol 1996;179:125.

\section{Supraregional Assay and Advisory Service}

Notification of Tenders Accepted by the SAAS Management Executive Board

In response to tenders received, the following laboratories have been designated to offer the Supraregional Assay and Advisory Service in the areas of expertise as follows:

\section{Uncommon Metabolic Thyroid Diseases}

Department of Clinical Biochemistry

Director: Dr P R Raggatt

Addenbrooke's Hospital NHS Trust, Hills Road, Cambridge CB2 2 QQ

Tel: 01223 217156; fax: 01223216862

\section{Metabolic Bone Disease}

Department of Clinical Biochemistry

Director: Professor C P Price

St Barts \& Royal London School of Medicine \& Dentistry, Turner Street, London E1 2AD

Tel: 01713777241 ; fax: 01713771544

Department of Chemical Pathology

Director: Dr Trevor Gray

Northern General Hospital NHS Trust, Herries Road, Sheffield S5 7AU

Tel: 0114243 4343; fax: 01142560472

Department of Clinical Chemistry

Director: Dr William D Fraser

Royal Liverpool University Hospital, Prescot Street, Liverpool L7 8XP

Tel: 0151706 4257; fax: 01517065806

Department of Chemical Pathology

Director: Dr Angela Fairney

St Mary's Hospital, Praed Street, London W2 1NY

Tel: 0171725 1097; fax: 01717251097

The standard of responses to the invitations to tender were very high, and the Supraregional Assay and Advisory Service Board compliment all the laboratories who bid for these assays on the quality of their presentations.

The Board are currently soliciting tenders for the supply of Assay and Advisory Services in the area of Porphyrin Metabolism. Enquires about this area may be made from the Chairman, Professor Vincent Marks, at the following address:

Professor Vincent Marks

Dean of Medicine

Chairman, SAAS Management Executive Board EIHMS, University of Surrey

Stirling House Campus

Surrey Research Park

Guildford GU2 5RF 